\title{
Diseño y estudio de resultados de una evalua- ción estructurada en un curso de Matemática con estudiantes que ingresan a la universidad
}

Autores: Gustavo Carnelli, Lucas Catalano, Alberto Formica e-mail:gcarnell@ungs.edu.ar;

lcatalano@ungs.edu.ar; aformica@ungs.edu.ar

Dirección: Juan María Gutierrez 1150 - C.P. 1613 -

Los Polvorines, Pcia de Bs. As., Argentina

Lugar de trabajo: Instituto del Desarrollo Humano, Universidad Nacional de General Sarmiento

Teléfono: (011) 4469-7701/2/3

\section{Resumen}

El trabajo que presentamos es un estudio sobre evaluación de aprendizajes en matemática en la instancia de ingreso a la Universidad Nacional de General Sarmiento. Los distintos equipos de coordinación del Taller de Matemática han implementado diversas acciones tendientes a mejorar los resultados del examen final, los cuales han tenido resultados históricamente bajos. Con la intención de obtener información más precisa sobre la disposición de las habilidades básicas que se trabajan en el curso, diseñamos una prueba de tipo estructurada, con ítems de selección múltiple y de verdadero-falso.

Presentamos una serie de características que deben reunir este tipo de ítems y mostramos el diseño de la prueba. Al aplicarla, obtuvimos 226 pruebas escritas sobre las que realizamos el análisis de las respuestas. Pudimos reconocer algunas fortalezas, como la aplicación del teorema de Pitágoras y la obtención de información a partir de un gráfico simple, así como debilidades en el cálculo de la pendiente de una recta y en algunas cuestiones del campo de lo algebraico.

Los resultados del análisis nos permiten realizar sugerencias a los docentes que dictan el curso con vistas a lograr aprendizajes de mayor calidad en los estudiantes.

Palabras clave: matemática, evaluación, aprendizaje en matemática. 


\begin{abstract}
The present paper is a research of learning in Mathematics at admission in the Universidad Nacional de General Sarmiento. Coordinating teams from Math's Workshop have implemented several actions in order to improve results in final exams, which have usually had bad results. A structured exam with multiple-choice and true - false items has been developed to obtain accurate information about basic competences worked during mathematical course. We present the design of the exam and several characteristics required to create each item it included. Once it applied we analyzed the answers obtained from 226 written tests. We were able to recognize either pupil's strengths as the application of the Pythagorean theorem and the collection of information from graphs or pupil's weaknesses as calculating the gradient of a line and some algebraically issues.

Derived data let us perform suggestions to teachers who dictate the course in order to achieve higher quality learning in students.
\end{abstract}

Keywords: mathematics, evaluation, maths learning. 


\section{Introducción}

Uno de los asuntos que más atraen el interés y la preocupación de los docentes y de quienes investigan sobre la enseñanza es la evaluación de los aprendizajes de los estudiantes. En este trabajo nos ocupamos de ello en el marco de la enseñanza de la matemática y en el nivel del ingreso a los estudios universitarios. Más concretamente, nos ubicamos en la instancia de examen final de la asignatura Taller de Matemática del Curso de Aprestamiento Universitario de la Universidad Nacional de General Sarmiento, ubicada en el conurbano bonaerense. La asignatura es común a todos los aspirantes a realizar carreras de grado y pregrado en la institución, es cuatrimestral y cuenta con una matrícula que varía entre 1000 y 2000 estudiantes por cuatrimestre. Los estudiantes que han obtenido la regularidad durante la cursada pero no han alcanzado la promoción, esto es, que han obtenido un promedio mayor o igual que 4 y menor que 7 , deben rendir examen final para completar la aprobación. Al respecto, cabe señalar que la evaluación de la asignatura incluye dos evaluaciones parciales y un informe escrito de carácter técnico acerca de alguna situación de interés general que pueda ser estudiada mediante contenidos matemáticos trabajados en el curso, como el análisis de los subsidios aplicados a las tarifas de servicios públicos. Esto significa que la propuesta combina aspectos de la evaluación sumativa, esto es, de balance de objetivos, realizada en momentos intermedios y/o finales y que apuntan a la certificación, con algunos elementos de la evaluación formativa (Camilloni, 2004), ya que pretende que el estudiante pueda analizar la situación en cuanto a su aprendizaje, reorientándolo o reencauzándolo con indicaciones del docente (estas nociones pueden verse y ampliarse en Camilloni, Basabe y Feeney, 2009, Gálvez Paredes, 2007 y Zanabria, 2014).

Todas las instancias de evaluación mencionadas, excepto el examen final, son administradas por el profesor a cargo del curso. En cambio, el examen final es administrado por el grupo de profesores y el equipo de coordinación de la asignatura a todo el conjunto de estudiantes que debe rendir el examen. Como dijimos, el examen final del Taller de Matemática es una instancia que deben rendir los estudiantes que han regularizado la asignatura pero no han logrado reunir las condiciones necesarias para su promoción directa. Esta evaluación es escrita e individual $y$, hasta la implementación del diseño que presentamos en este trabajo, consistía en una serie de cinco o seis actividades que evaluaban los distintos contenidos de la asignatura. En ese esquema de examen final se requiere la presentación escrita de todos los procedimientos efectuados y justificaciones necesarias para su resolución. Las actividades conjugan el uso de distintos procedimientos (aritméticos, algebraicos, geométricos, etc.) con la explicitación de argumentos que justifiquen su uso o necesidad y de estilo similar a las propuestas en cada una de las evaluaciones parciales. No obstante haber reunido las condiciones de regularidad durante el cursado de la asignatura, el rendimiento de los estudiantes en el examen final ha sido históricamente bajo (usualmente, entre un 20 y un $35 \%$ de aprobados). Cabe mencionar que el examen final se rinde alrededor de dos semanas después de finalizada la cursada.

Los equipos de coordinación de la asignatura han implementado diversas acciones tendientes a mejorar los resultados. Entre ellas, una mayor conexión entre el formato y contenido del examen final y de la última evaluación parcial realizada durante la cursada y la implementación de una clase adicional de preparación específica del examen final. Sin embargo, todo esto impactó muy poco en la tasa de aprobación.

En el contexto mencionado, nos propusimos diseñar un instrumento distinto al usual, de modo de obtener información más precisa acerca de la disposición de las habilidades básicas que se trabajan en el curso. Con esto, podríamos pensar en dar orientaciones para la enseñanza de modo de favorecer aprendizajes de mayor calidad en los estudiantes. Para precisar lo que entendemos por habilidades básicas, podemos tomar las habilida- 
des de rango bajo y medio propuestas por Jiménez Rodríguez (1997), quien, al respecto, considera: «las habilidades de bajo rango, como usar rutinas técnicas, algoritmos estándares, dar definiciones, resolver ejercicios tipo, recordar hechos aislados, etc.» $\mathrm{y}$ «las habilidades de rango medio, como resolver problemas tipo, hacer conexiones simples, relacionar, integrar, etc.».

\section{La evaluación y los instrumentos para evaluar los aprendizajes}

Evaluar los aprendizajes de los estudiantes es una compleja actividad que atañe a la enseñanza. Para ello, es necesario disponer de información sistemática sobre el aprendizaje de los estudiantes. Los resultados de la evaluación no solo sirven para decidir sobre el nivel alcanzado por el estudiante sino también para regular y orientar los procesos de enseñanza y de aprendizaje.

La recolección de información puede hacerse utilizando distinto tipo de instrumentos. Los más difundidos en el sistema educativo son las pruebas y los instrumentos de observación (como las listas de cotejos y las rúbricas). Las pruebas pueden ser formales, que son aquellas diseñadas por especialistas para ser administradas a amplias poblaciones (por ejemplo, operativos de evaluación de alcance nacional) o no formales, que son las diseñadas y administradas por el docente en sus cursos. De acuerdo con el modo de resolución, las pruebas pueden ser orales o escritas. Las pruebas escritas pueden clasificarse en dos grandes grupos: objetivas (o estructuradas) y subjetivas (o de composición) (Varela, Guasco, Gerompini y Martello, 1996). A modo de contrapunto entre ambos tipos y a grandes rasgos, podemos decir que en las pruebas objetivas la opinión del corrector no tiene incidencia, mientras que en las subjetivas esta es mayor. En las pruebas objetivas, los ítems admiten respuestas únicas, mientras que en las otras las respuestas admiten distintos grados de calidad; la correcta interpretación de las consignas es más decisiva en las objetivas, mientras que la mejor expresión lo es en las subjetivas. Por último, las objetivas tienen un diseño complejo y una corrección simple, situación que parcialmente se invierte en las subjetivas. Por sobre todo, en términos de lo que se pretende del estudiante, las objetivas privilegian la selección e identificación y las subjetivas, la producción.

Algunos autores, como Varela, Guasco, Gerompini y Martello (1996), distinguen dos tipos de pruebas escritas subjetivas: las de respuesta restringida y las de ensayo. Las primeras son aquellas en las que la elaboración personal es mediana o baja, con consignas focalizadas. Por su parte, las pruebas de ensayo tienen consignas más abiertas y la elaboración personal es alta.

Un tipo de pruebas diferente son las alternativas o portafolios, en los que el estudiante reúne trabajos durante un lapso, realiza una selección de ellos e identifica sus progresos en el aprendizaje. Este instrumento, según Danielson y Abrutyn (1999), favorece los procesos metacognitivos de reflexión y autoevaluación del estudiante.

\subsection{Las pruebas objetivas}

Entendemos que las pruebas objetivas son instrumentos apropiados para evaluar habilidades simples, además de poder barrer un número amplio de asuntos de los cuales interesa detectar, principalmente, la capacidad de identificar, de seleccionar, de recordar definiciones y propiedades y de realizar procedimientos cortos. Además, son un medio directo y simple de obtener resultados aplicados a cantidades grandes de estudiantes, minimizan la subjetividad del evaluador y son de rápida corrección. Debe atenderse que no son adecuadas para evaluar procesos complejos de pensamiento 0 procedimientos largos (sobre esto, puede ampliarse en Cols, Amantea y Guervitz, 2007).

Los tipos de ítems usuales en las pruebas objetivas son los siguientes: de selección múltiple, verdadero-falso, de emparejamiento y de ordenamiento. A partir de lo desarrollado en Varela, Guasco, Ge- 
rompini y Martello (1996), consideramos que una prueba estructurada, en cuanto a su diseño:

- Requiere mayor atención en su elaboración, para evitar ambigüedades e imprecisiones que puedan confundir al estudiante.

- Debe contener varios ítems, para minimizar la influencia de las respuestas por azar.

- Debe contener ítems independientes unos de otros para evitar el arrastre de error en las respuestas.

- Tiene que contener respuestas correctas únicas que previamente estén determinadas y deben ubicarse en los distintos ítems de modo que no quede sugerido un patrón de ubicación.

Un ítem estructurado:

- No requiere justificación de la respuesta ya que, si se pidiera el ítem dejaría de ser estructurado.

- No debe incluir pistas que puedan sugerir o hacer evidente la respuesta.

- Tiene que estar formulado de modo conciso, claro y afirmativo.

- Debe evaluar el conocimiento de asuntos significativos y no detalles menores.

- No tiene que incluir términos ambiguos, como «frecuentemente», «en gran medida», etcétera.

- No debe incluirse información no pertinente.

\subsection{1. İtems de emparejamiento}

Son aquellos en los que hay que poner en correspondencia los elementos de una lista con los de otra de acuerdo con algún criterio. Por ejemplo:

İtem: en la lista de la izquierda se da una serie de funciones y en la de la derecha una serie de conjuntos. Unir con flechas los casos en que la función de la izquierda tiene por conjunto de positividad al conjunto de la derecha.

\begin{tabular}{|l|c|}
\hline Función & Conjunto de positividad \\
\hline$f: R \rightarrow R / f(x)=x^{2}$ & $\mathrm{R}$ \\
$f: \mathrm{R} \rightarrow \mathrm{R} / f(x)=x^{3}$ & $\mathrm{R}-\{0\}$ \\
$f: \mathrm{R} \rightarrow \mathrm{R} / f(x)=-2 x$ & $(0,+\infty)$ \\
$f: \mathrm{R}-\{0\} \rightarrow \mathrm{R} / f(x)=1 / x$ & $(-\infty, 0)$ \\
\hline
\end{tabular}

\subsection{2. Ítems de verdadero o falso}

Son enunciados en los que hay que decidir acerca de su veracidad y responder Verdadero o Falso. Debe formularse de modo que sea inequívocamente verdadero o falso.

Como ya dijimos, el diseño de ítems estructurados es delicado. Por ejemplo, cuando se plantea una afirmación como: 303 es múltiplo de 11 y 7 es divisor de -7, es importante observar que no solo se evalúan conocimientos de divisibilidad sino que también se evalúa cuándo es verdadero y cuándo es falso un enunciado que incluye una conjunción. Un ejemplo de este tipo de ítem de verdadero o falso que no contiene esta dificultad que acabamos de mencionar es el siguiente:

İtem: decidir si el enunciado a continuación es verdadero ofalso:

«Todas las funciones cuadráticas tienen dos raíces reales.»

\subsection{3. Ítems de selección múltiple}

Están compuestos por un cuerpo formado por una pregunta o frase incompleta y un conjunto de opciones de respuesta (la correcta y los distractores). Consideramos que este tipo de ítem debe reunir las siguientes características:

- Poder anticiparse la naturaleza de la respuesta aun no si no se ven las alternativas.

- Solo una de las alternativas debe ser correcta o puede preguntarse por la mejor respuesta, especificando con claridad esa condición.

- No incluir distractores absurdos o no homogéneos con el resto.

- Evitarse incluir como alternativa «ninguna de las anteriores es correcta» 0 "todas las anteriores son correctas» ya que estas generan incertidumbre y no garantizan unicidad de la respuesta.

Seguidamente, analizamos algunos ejemplos con defectos de formulación.

İtem: el resultado del cálculo

$\left(\frac{1}{2}+3\right)^{-1}-2^{2} \cdot 4^{1 / 2} \quad$ es... 

A) $-54 / 7$
B) $-23 / 2$
C) $-52 / 7$
D) $-24 / 3$

La alternativa $A$ es la única correcta; la alternativa B se obtiene si la potencia de exponente negativo se resuelve cambiando el signo de la base; la $\mathrm{C}$ surge de resolver primero la resta y luego el producto; la $D$ resulta de resolver la potencia con exponente negativo invirtiendo a cada una de las fracciones que figuran en el paréntesis y luego realizando la suma. Si, por ejemplo, un estudiante resolviera la potencia con exponente fraccionario de algún modo incorrecto o incurriera en otro error de operatoria distinto de los contemplados, no encontraría la opción correspondiente y se vería inducido a elegir por azar. Puede considerarse que este problema se soluciona incluyendo como opción «ninguna de las anteriores es correcta». Sin embargo, al incluirse esta alternativa, el resto de las opciones deja de ser determinante, con márgenes de incertidumbre (entre esas alternativas puede estar la correcta o no estar). Además, si un estudiante eligiera la opción «ninguna de las anteriores», la única información que puede obtenerse de sus conocimientos es que no logra resolver en forma correcta el cálculo pero es factible saber cuál es el error cometido, con el riesgo de que este no sea importante.

\section{Diseño de una prueba estructurada: el examen final del Taller de Matemática}

El curso de Taller de Matemática de la instancia de ingreso a la Universidad Nacional de General Sarmiento aborda los temas: Números y cálculos, Perímetro y Área, Proporcionalidad y Geometría, Álgebra, Modelización, Modelización con funciones lineales y Modelización con funciones cuadráticas. Diseñamos el examen final de tipo estructurado de manera de que la totalidad de los temas tuviera presencia con un peso compatible con los tiempos que invierten en la cursada y que incluyera ítems de selección múltiple y de verdadero-falso. La prueba completa, que puede verse en el Anexo, constó de 12 ítems de selección múltiple y 4 de verdadero-falso.

Debido a que los estudiantes que rindieron esta prueba están en el primer cuatrimestre de cursada de sus estudios superiores y a que la prueba es de un tipo distinto de las usuales, para familiarizarlos con los ejercicios propusimos que los docentes trabajaran en las últimas clases del cuatrimestre algunos ítems estructurados. Además, dictamos una clase destinada a la totalidad de estudiantes que debía rendir el examen, y allí se trabajaron ítems estructurados haciendo hincapié tanto en el contenido de referencia como en el modo de abordarlos.

Ejemplificamos el criterio con el cual diseñamos los ítems con un par de ellos.

Entre los ítems de selección múltiple, propusimos el siguiente:

Ittem: el perímetro del semicírculo de la derecha, de radio $3 \mathrm{~cm}$, aproximado con dos cifras decimales, es...

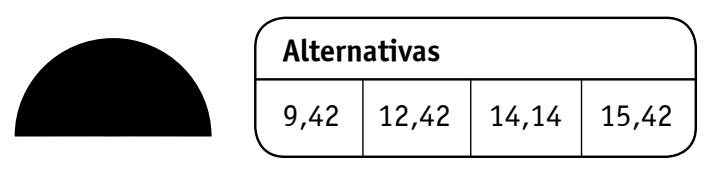

La respuesta correcta es la última. Los distractores fueron pensados del siguiente modo:

9,42 es la respuesta que se obtiene si se omite sumar el diámetro al resultado de $\frac{\pi .6}{2}$;

12,42 es la respuesta obtenida al sumar el radio en vez del diámetro al resultado de $\frac{\pi .6}{2}$

y 14,13 es la respuesta obtenida si se calculase el área de la figura en vez del perímetro.

Como ítem de verdadero-falso propusimos:

İtem: decidir si cada afirmación es verdadera ofalsa. Indicarlo con $V$ o $F$ en los recuadros.

Dada la función $f: R \rightarrow R / f(x)=2 x^{2}$ se cumple que... 


\begin{tabular}{|l|l|l|}
\hline & V & F \\
\hline el punto $(-1 ;-2)$ pertenece a su gráfico & & \\
\hline en el intervalo $(-\infty, 0)$ es decreciente & & \\
\hline no tiene raíces reales & & \\
\hline el vértice de la parábola es el punto $(0 ; 2)$ & & \\
\hline
\end{tabular}

El ítem contiene cuatro enunciados independientes que giran en torno de una cierta función dada y barren varias de las características de las funciones que se estudian en el curso.

En la prueba completa hemos incluido algún caso en que el enunciado está formulado por la negativa, contrario a la indicación mencionada como característica de los ítems estructurados. Sin embargo, entendemos que en los casos propuestos es pertinente hacerlo en función de las características del contenido de referencia. Por ejemplo, referido al ítem último, el hecho que una función no tenga ceros es un asunto de interés y tratamiento específico. Vale aclarar que el criterio de aprobación del examen, que está incluido en el enunciado completo de la prueba que se exhibe en el Anexo, no es tenido en cuenta en este trabajo ya que el interés está puesto en las respuestas dadas a cada uno de los ítems y no en los niveles de aprobación.

\section{Análisis de los resultados de la prueba estructurada}

\subsection{Resultados globales}

De esta prueba recogimos 226 exámenes respondidos. Algunos datos generales de los resultados, que permiten conocer las características más globales del conjunto de datos que analizamos, son:

- Diagrama de barras que muestra la distribución de los 226 estudiantes según cuántos de los 16 ítems respondieron correctamente (Figura 1 ).

- Media del conjunto de datos (cantidad de aciertos promedio): 5,54.

- Mediana del conjunto de datos (cantidad de aciertos que divide a los 226 en dos mitades): 5.

- Moda del conjunto de datos (cantidad de aciertos más repetida): 5 .

A partir de lo anterior, podemos decir que no ha habido un grupo numeroso de estudiantes que haya respondido bien a casi todas las preguntas ni tampoco que haya contestado mal casi todas.

\subsection{Resultados por ítem}

Exhibimos a continuación los enunciados de los ítems que conformaron la prueba, una síntesis de

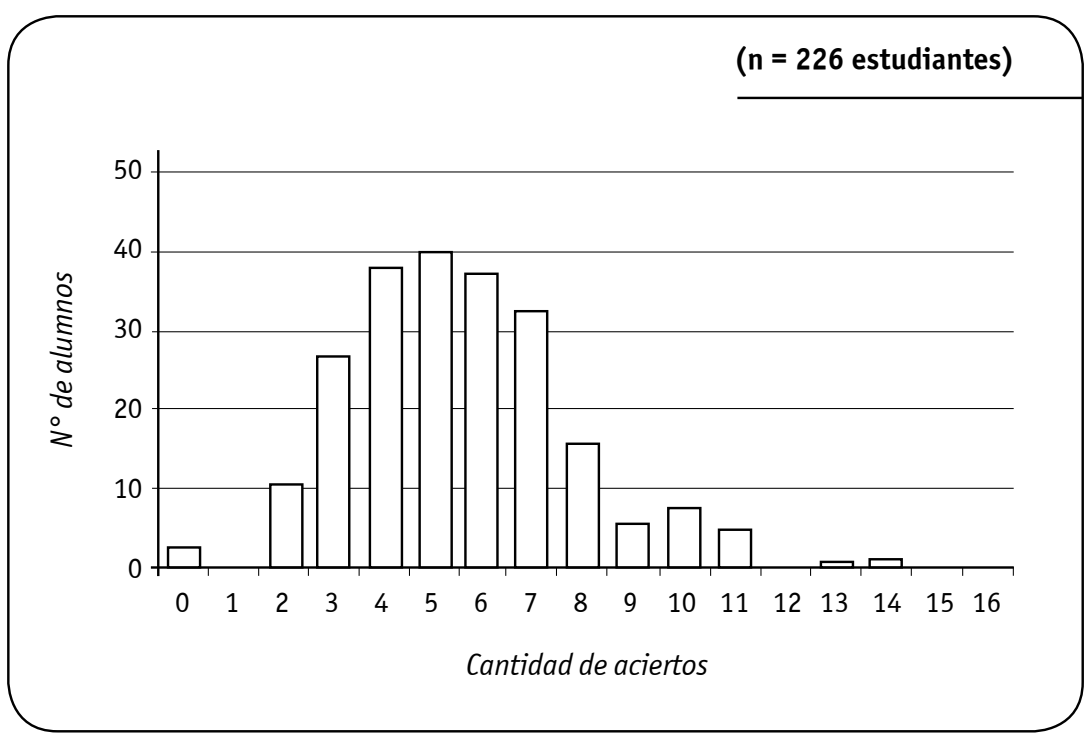

Figura 1.

Distribución de la cantidad de aciertos en la prueba. 
las respuestas de los estudiantes y una consideración sobre las mismas. En la tabla que se presenta para cada ítem, la primera fila contiene las alternativas que se propusieron y la segunda indica el porcentaje de los 226 estudiantes que eligió esa alternativa. La última columna $(\mathrm{S} / \mathrm{C})$ contempla los casos en que el ítem no fue respondido. Los ejercicios 1 a 12 son los de selección múltiple, mientras que los del 13 al 16 son los de verdadero-falso.

İtem 1: el número racional que se señala con $\mathrm{x}$ en la recta numérica es...

\begin{tabular}{l|l|l}
\hline & & \\
2 & $x$ & 3
\end{tabular}

\begin{tabular}{|l|l|l|l|l|l|}
\hline Alternativas & $2 / 3$ & $7 / 3$ & 2,6 & 2,75 & $\mathrm{~S} / \mathrm{C}$ \\
\hline$\%$ de Respuestas & 25 & 6 & 27 & 30 & 12 \\
\hline
\end{tabular}

Solo un poco más de la cuarta parte de los estudiantes da la respuesta correcta. Una cantidad similar opta por un resultado que puede haber sido pensado como una aproximación al valor indicado en la recta 0 también como que el entero está dividido en 4 partes y no en 3. Otra cantidad parecida da una respuesta que sería válida si los enteros indicados fueran 0 y 1 , hecho que es frecuente en este tipo de actividades.

İtem 2: un número racional cuyo desarrollo decimal NO es finito es...

\begin{tabular}{|l|c|c|c|c|c|}
\hline Alternativas & $-8 / 7$ & $-3 / 4$ & $\sqrt{7}$ & $25 / 8$ & $\mathrm{~S} / \mathrm{C}$ \\
\hline \% de Respuestas & 23 & 13 & 29 & 17 & 18 \\
\hline
\end{tabular}

Las respuestas dadas se reparten de una forma más o menos pareja, lo cual dificulta la posibilidad de inferir algo más que el bajo nivel de conocimiento que mostró el grupo evaluado respecto del desarrollo decimal de un número racional. Podemos precisar, además, que alrededor de un $30 \%$ de los estudiantes no reconoce un número irracional cuando está expresado mediante un radical.
Ítem 3: a un precio se lo aumenta en un $10 \%$ y al precio obtenido se lo aumenta en otro $10 \%$. El porcentaje total de aumento (del precio inicialal precio final) es...

\begin{tabular}{|l|c|c|c|c|c|}
\hline Alternativas & $10 \%$ & $15 \%$ & $20 \%$ & $21 \%$ & $\mathrm{~S} / \mathrm{C}$ \\
\hline$\%$ de Respuestas & 15 & 2 & 41 & 35 & 8 \\
\hline
\end{tabular}

Las 3/4 partes de las respuestas se concentra, al sumarse, en el resultado correcto y el error más frecuente. Además, 4 de cada 10 estudiantes no distingue aumentos acumulados de aumentos no acumulados, mientras que sí lo distingue una cantidad algo menor.

İtem 4: la concentración de un cierto componente en un producto es $0,2 \mathrm{mg} / 3 \mathrm{~g}$. Si un paciente debe realizar una ingesta diaria de $5 \mathrm{mg}$ de ese componente, la cantidad de producto que debe tomar por día (en gramos) es...

\begin{tabular}{|l|c|c|c|c|c|}
\hline Alternativas & 0,0033 & 0,33 & 75 & 75.000 & $\mathrm{~S} / \mathrm{C}$ \\
\hline \% de Respuestas & 2 & 33 & 42 & 1 & 22 \\
\hline
\end{tabular}

Algo más que 4 de cada 10 estudiantes interpreta correctamente la situación de proporcionalidad, mientras una cantidad menor confunde las variables. Asimismo, casi una cuarta parte de los estudiantes no responde el ítem.

İtem 5: si los catetos de un triángulo rectángulo miden $\sqrt{5} \mathrm{~cm}$ y $5 \mathrm{~cm}$, el tercer lado mide...

\begin{tabular}{|l|c|c|c|c|c|}
\hline Alternativas & $\sqrt{10}$ & $\sqrt{20}$ & $\sqrt{30}$ & 30 & $\mathrm{~S} / \mathrm{C}$ \\
\hline$\%$ de Respuestas & 10 & 4 & 60 & 22 & 4 \\
\hline
\end{tabular}

El cálculo del tercer lado de un triángulo rectángulo conocidos los catetos resultó ser uno de los conocimientos que más estudiantes pudieron mostrar en toda la prueba. Casi la cuarta parte usa que la suma de los cuadrados de los catetos da el valor de la hipotenusa y no de su cuadrado. 
Ítem 6: la figura es un semicírculo de radio $3 \mathrm{~cm}$. Su perímetro (en $\mathrm{cm})$, aproximado con dos cifras decimales, es...

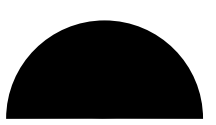

\begin{tabular}{|l|c|c|c|c|c|}
\hline Alternativas & 9,42 & 12,42 & 14,14 & 15,42 & $\mathrm{~S} / \mathrm{C}$ \\
\hline \% de Respuestas & 54 & 3 & 11 & 23 & 9 \\
\hline
\end{tabular}

Solo la cuarta parte de los estudiantes calcula correctamente el perímetro. Algo más de la mitad de los estudiantes no suma el diámetro, considerando solo el valor de la semicircunferencia como respuesta.

Ítem 7: el conjunto solución de la ecuación $x^{2}+x=0$ es $S=\ldots$

\begin{tabular}{|l|c|c|c|c|c|}
\hline Alternativas & $\phi$ (vacío) & $\{0\}$ & $\{0,1\}$ & $\{0,-1\}$ & S/C \\
\hline \% de Respuestas & 34 & 24 & 7 & 23 & 12 \\
\hline
\end{tabular}

Nuevamente, solo la cuarta parte encuentra el conjunto solución correcto. Otro tanto halla solo una de las dos soluciones. Además, la tercera parte sostiene que la ecuación no tiene solución aunque no es posible inferir lo que estos estudiantes han realizado para elegir esa alternativa, quizás algún error de operatoria. No obstante, estos estudiantes no han podido considerar que el 0 es una solución, lo que es fácil de ver por simple inspección de la ecuación, o de sospechar por la lectura de las alternativas.

İtem 8: una expresión algebraica que NO es equivalente a $4 n-4$ es...

\begin{tabular}{|c|c|c|c|c|c|}
\hline Altern. & $(2 n+2) \cdot(2 n-2)$ & $2 n+2 .(n-2)$ & $n^{2}-(n-2)^{2}$ & $(n-1) \cdot 4$ & $\mathrm{~S} / \mathrm{C}$ \\
\hline \% de R. & 28 & 7 & 46 & 12 & 7 \\
\hline
\end{tabular}

Este ítem presenta otra vez una cantidad de aciertos de alrededor de la cuarta parte de los estudiantes. Casi la mitad elige una alternativa en la que probablemente hayan incurrido en errores de operatoria o bien hayan descartado por su apariencia de expresión cuadrática.

Ítem 9: si una ecuación cuadrática tiene dos soluciones reales distintas, entonces un valor que puede tomar su discriminante es...

\begin{tabular}{|l|c|c|c|c|c|}
\hline Alternativas & -10 & $-0,5$ & 0 & 0,5 & $\mathrm{~S} / \mathrm{C}$ \\
\hline \% de Respuestas & 16 & 8 & 29 & 29 & 17 \\
\hline
\end{tabular}

Una vez más, la cantidad de respuestas correctas ronda la cuarta parte. La otra opción más elegida corresponde al caso de solución única, lo que evidencia desconocimiento de la propiedad del discriminante de una ecuación cuadrática.

Ítem 10: dados los puntos $(3 ;-1)$ y $(-4 ;-5)$, la pendiente de la recta que los contiene vale...

\begin{tabular}{|l|c|c|c|c|c|}
\hline Alternativas & $-6 / 7$ & $-4 / 7$ & $4 / 7$ & $6 / 7$ & S/C \\
\hline$\%$ de Respuestas & 12 & 20 & 9 & 17 & 42 \\
\hline
\end{tabular}

El cálculo de la pendiente de una recta conocidos dos de sus puntos en un gráfico resultó ser el respondido correctamente por menos estudiantes, como también fue el ítem que más estudiantes dejaron sin contestar. Más allá de que se evalúa el conocimiento del cálculo de la pendiente a partir de dos puntos y también la operatoria con enteros, es importante observar que más de un $40 \%$ de los estudiantes no responde al ítem y apenas 1 de cada 10 lo hace correctamente. 
Ítem 11: el gráfico que sigue muestra la distancia a la que se encuentra Juan de su casa durante una jornada de trabajo.

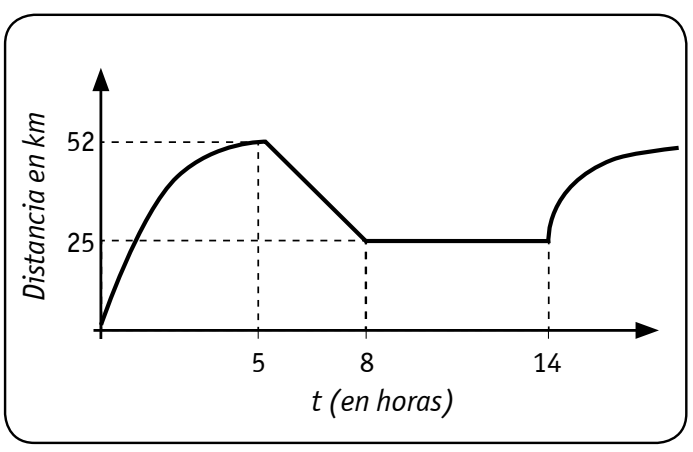

La cantidad de veces que Juan estuvo a $30 \mathrm{~km}$ de su casa es...

\begin{tabular}{|l|c|c|c|c|c|}
\hline Alternativas & 1 & 2 & 3 & 4 & S/C \\
\hline \% de Respuestas & 4 & 13 & 77 & 0 & 6 \\
\hline
\end{tabular}

Este ítem es el que tuvo el mejor porcentaje de respuestas correctas. Más de las tres cuartas partes resolvió correctamente la lectura de una gráfica simple.

Ítem 12: teniendo en cuenta el gráfico del Ítem $11 \mathrm{y}$ que la variación de la distancia en el intervalo $(5,8)$ es lineal, la distancia a la que se encuentra Juan de su casa a las 6 horas es (en km)...

\begin{tabular}{|l|c|c|c|c|c|}
\hline Alternativas & 9 & 43 & 45 & 51 & S/C \\
\hline$\%$ de Respuestas & 3 & 29 & 37 & 19 & 12 \\
\hline
\end{tabular}

Nuevamente, las respuestas correctas rondaron la cuarta parte. La concentración de respuestas elegidas permite suponer que muchos alumnos propusieron una respuesta aproximada.

Ítems 13 a 16: Dada la función $f: \mathrm{R} \rightarrow \mathrm{R} / f(x)=2 x^{2}$ se cumple que...

\begin{tabular}{|c|c|c|c|c|}
\hline & $\mathbf{V}$ & $\mathbf{F}$ & $\mathrm{N} / \mathrm{C}$ \\
\hline & & \multicolumn{3}{|c|}{$\%$ de Respuestas } \\
\hline 13 & $\begin{array}{l}\text { el punto }(-1 ;-2) \text { pertenece } \\
\text { a su gráfico }\end{array}$ & 19 & 43 & 38 \\
\hline 14 & $\begin{array}{l}\text { en el intervalo }(-\infty, 0) \text { es } \\
\text { decreciente }\end{array}$ & 30 & 34 & 36 \\
\hline 15 & no tiene raíces reales & 32 & 34 & 34 \\
\hline 16 & $\begin{array}{l}\text { el vértice de la parábola es } \\
\text { el punto }(0 ; 2)\end{array}$ & 43 & 20 & 37 \\
\hline
\end{tabular}

Una mirada global a los 4 ítems permite suponer un bajo conocimiento de las características de una función cuadrática sencilla. Esta idea se refuerza en el hecho de que el mayor porcentaje de aciertos se da en la decisión acerca de si un punto pertenece a su gráfico, que es el único ítem que no requiere saber las especificidades de las funciones cuadráticas. También sucede que, en los 4 ítems, alrededor de la tercera parte de los estudiantes no responde.

\section{Conclusiones}

La principal intención que tuvimos con la implementación de este tipo de examen fue conocer con más precisión el nivel de aprendizaje de las habilidades básicas del curso en los estudiantes que rinden examen final. En este sentido, los resultados de la prueba permiten obtener algunas conclusiones aunque menos de las que esperábamos. En cuanto a los asuntos que más estudiantes demostraron saber, encontramos la obtención del tercer lado de un triángulo rectángulo conocidos los catetos y la obtención de preimágenes en una función dada por su gráfico en una situación expresada en lenguaje coloquialy sin uso de notación simbólica de funciones.

Entre las cuestiones que menos estudiantes demostraron conocer está la pendiente de la recta. Eso fue observado en dos instancias: cuando se pidió el cálculo de la pendiente dados dos puntos y también en el cálculo de una imagen en una función dada por un gráfico y cuya obtención requería el conocimiento de la pendiente. Asimiso, fue muy pobre el conocimiento exhibido sobre el tipo de desarrollo decimal de los números. Fuera de lo señalado, los 
resultados muestran poca consolidación de los conocimientos evaluados.

En el terreno de lo numérico, es necesario revisar la representación de los racionales en la recta numérica y la distinción entre racionales e irracionales dados en sus distintas formas.

En el campo del álgebra, tanto la resolución de ecuaciones cuadráticas como el reconocimiento de expresiones algebraicas equivalentes mostraron bajos resultados. Resulta necesario revisar la noción de solución y, en particular, la posibilidad de saber si un número es solución o no de una ecuación sin resolverla. Sobre la noción de perímetro de una figura, podemos decir que los estudiantes disponen del conocimiento de la fórmula para la obtención del perímetro de una circunferencia pero no realizan correctamente su cálculo en una figura cuyo perímetro se compone de dos figuras simples. Es alta la cantidad de estudiantes que aplica una estrategia de resolución válida para el área pero no para el perímetro.
Respecto de la proporcionalidad directa, es necesario revisar la noción de aumentos acumulados. Las funciones cuadráticas son el último tema en la programación de la materia. Es evidente que debe invertirse más tiempo en su enseñanza.

Los indicadores globales del examen desarrollados más arriba muestran que las fortalezas y debilidades observadas no se concentran en ciertos individuos, lo que podría haberse sospechado al ver que varios ítems fueron resueltos correctamente por alrededor del $25 \%$ de los estudiantes, sino que las mismas se presentan en forma repartida.

Por último, y debido al escaso contacto con este tipo de pruebas, es posible que varios estudiantes no hayan realizado la prueba con la suficiente atención que estos ítems requieren y hayan incurrido en errores de distracción. Esto podría verse, por ejemplo, en el error de omitir la extracción de la raíz cuadrada a la suma de los cuadrados de los catetos en el teorema de Pitágoras.

\section{Referencias bibliográficas}

Camilloni, A. (1997). La calidad de los programas de evaluación y de los instrumentos que los integran. En Camilloni, A.; Celman, S.; Litwin, E. y Palou de Maté, M. (1997). La evaluación de los aprendizajes en el debate didáctico contemporáneo. Buenos Aires: Paidós.

--— (2004). Sobre la Evaluación Formativa de los aprendizajes. Quehacer Educativo, XIV(68). Montevideo

Camilloni, A.; Basabe, L. y Feeney, S. (2009). Los formatos de evaluación de los aprendizajes y sus relaciones con las modalidades de estudio de los alumnos universitarios. Consultado el 1 de diciembre de 2015 en http://www.ungs.edu.ar/cienciaydiscurso/?page_id=232

Cols, E.; Amantea, A. y Guervitz, M. (2007). Las prácticas de evaluación en el nivel medio: problemáticas y enfoques. Escuela de Capacitación Docente-CEPA. Secretaría de Educación del Gobierno de la Ciudad de Buenos Aires.

Cols, E.; Basabe, L. y Feeney, S. (2006, octubre). La evaluación escolar: de los lemas a los problemas. En 12(ntes), (8). Consultado el 15 de noviembre de 2015 en www.ungs.edu.ar/cienciaydiscurso/ wp-content/uploads/2011/11/Cols-Basabe-Feeney-2006.pdf

Danielson, C. y Abrutyn, L. (1999). Una introducción al uso de portafolios en el aula. México: Fondo de Cultura Económica.

Gálvez Paredes, R. (2007). Matemática. Serie 1 para docentes de secundaria. Fascículo 3: Evaluación en el área de Matemática. Lima: Ministerio de Educación.

Varela, L.; Guasco, M.; Gerompini, A. y Martello, S. (1996). Matemática - Metodología de la Enseñanza. Buenos Aires: Prociencia-Conicet.

Zanabria, C. (2014). ¿La evaluación de aprendizajes, una forma de enseñanza, una oportunidad de aprendizaje?: creencia y práctica. Una mirada desde la educación matemática. Santa Fe: Ediciones UNL. 


\section{Anexo}

Enunciado completo de la prueba tal como fue administrado

\begin{tabular}{|l|l|l|}
\hline CAU - TALLER DE MATEMÁTICA - EXAMEN FINAL - DICIEMBRE 2015 \\
\hline APELLIDO Y NOMBRE & \multicolumn{2}{|l|}{} \\
\hline DNI & TEMA 1 \\
\hline
\end{tabular}

Para aprobar es necesario: a) sumar 4 puntos y b) del total de 16 ítems que componen el examen, la cantidad de respuestas correctas debe ser mayor que la de incorrectas (no se contabilizan las que no se contestan). En cada uno de los ítems 1 a 12, elegir la única alternativa correcta. Marcarla con una cruz en el recuadro de la izquierda de la opción elegida. Cada ítem vale 0,75 puntos.

1. El número racional que se señala con $x$ en la recta numérica es...

\begin{tabular}{l|l|l}
\hline & 1 & 1 \\
\hline 2 & $\times$ & 3
\end{tabular}
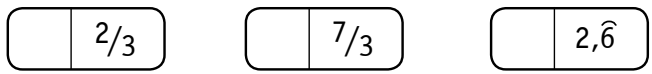

2. Un número que es racional y cuyo desarrollo decimal N0 es finito es...
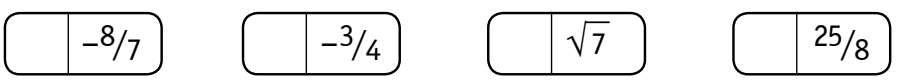

3. A un precio se lo aumenta en un $10 \%$ y al precio obtenido se lo aumenta en otro $10 \%$. El porcentaje total de aumento (del precio inicial al precio final) es...
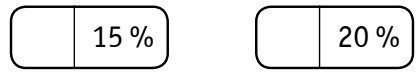

4. La concentración de un cierto componente en un producto es $0,2 \mathrm{mg} / 3 \mathrm{~g}$. Si un paciente debe realizar una ingesta diaria de $5 \mathrm{mg}$ de ese componente, la cantidad máxima de producto que debe tomar por día (en gramos) es...
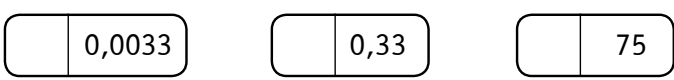

75.000

5. Si los catetos de un triángulo rectángulo miden $\sqrt{5} \mathrm{~cm}$ y $5 \mathrm{~cm}$, el tercer lado mide ...
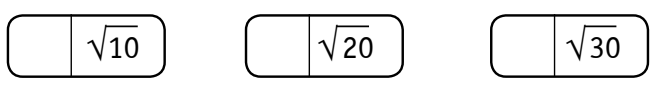

$$
30
$$


6. La figura es un semicírculo de radio $3 \mathrm{~cm}$. Su perímetro (en $\mathrm{cm}$ ), aproximado con dos cifras decimales, es...
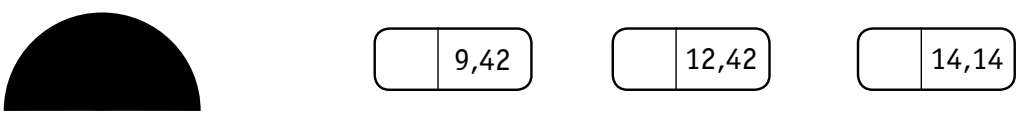

7. El conjunto solución de la ecuación $x^{2}+x=0$ es $S=\ldots$
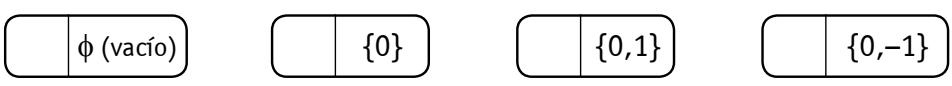

8. Una expresión algebraica que NO es equivalente a $4 n-4$ es...
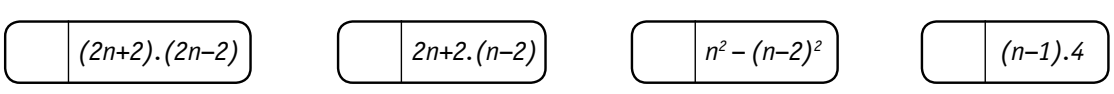

9. Si una ecuación cuadrática tiene dos soluciones reales distintas entonces, un valor que puede tomar su discriminante es...

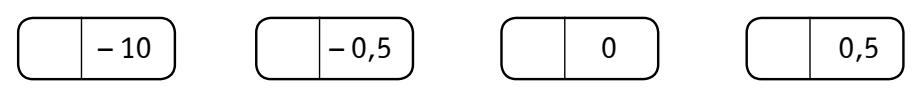

10. Dados los puntos $(3 ;-1)$ y $(-4 ;-5)$, la pendiente de la recta que los contiene vale...
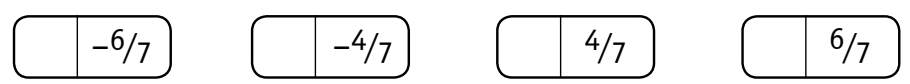

11. El gráfico que sigue muestra la distancia a la que se encuentra Juan de su casa durante un cierto tiempo.

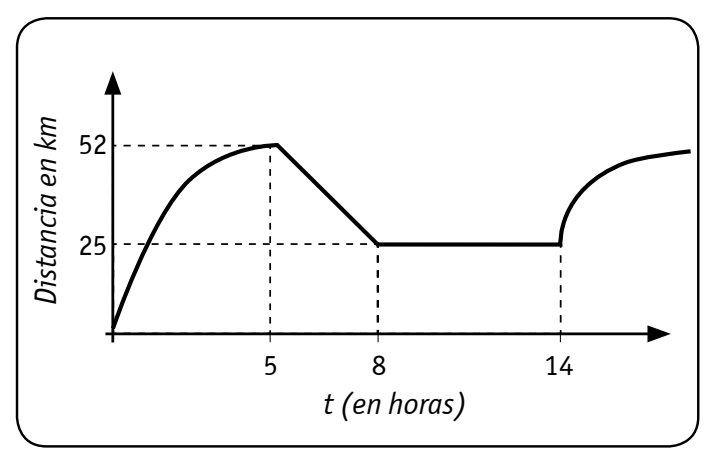

La cantidad de veces que Juan estuvo a $30 \mathrm{~km}$ de su casa es...
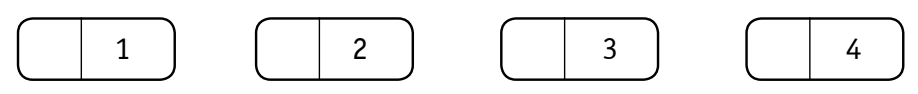
12. Teniendo en cuenta el gráfico del Ítem 11 y que la variación de la distancia en el intervalo $(5,8)$ es lineal, la distancia a la que se encuentra Juan de su casa a las 6 horas es (en km)...

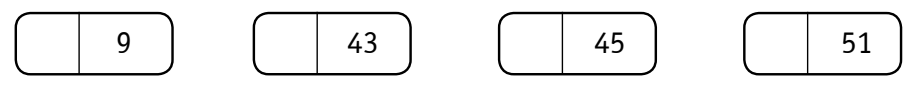

En los ítems 13 a 16, decidir si cada afirmación es verdadera o falsa. Indicarlo con $\mathrm{V}$ o $\mathrm{F}$ en los recuadros. Cada ítem vale 0,25 puntos.

13 a 16. Dada la función $f: R \rightarrow R / f(x)=2 x^{2}$ se cumple que...

\begin{tabular}{|l|l|l|}
13 & el punto $(-1 ;-2)$ pertenece a su gráfico & \\
14 & en el intervalo $(-\infty, 0)$ es decreciente & \\
15 & no tiene raíces reales & \\
16 & el vértice de la parábola es el punto $(0 ; 2)$ & \\
\hline
\end{tabular}

\section{PARA EL CORRECTOR:}

\begin{tabular}{|l|l|l|l|l|}
\hline Respuestas correctas & & Sin contestar & \multirow{2}{*}{ NOTA: } \\
\hline Respuestas incorrectas & & $\begin{array}{l}\text { ¿Más correctas } \\
\text { que incorrectas? }\end{array}$ & & \\
\hline
\end{tabular}

